\title{
Primary Biliary Cirrhosis-Autoimmune Hepatitis Overlap Syndrome Concomitant with Systemic Sclerosis, Immune Thrombocytopenic Purpura
}

\author{
Megumi Toyoda ${ }^{1}$, Hiroaki Yokomori ${ }^{1}$, Fumihiko Kaneko ${ }^{1}$, Hide Yoshida ${ }^{2}$, Kenta Hoshi ${ }^{2}$, \\ Hajime Takeuchi ${ }^{1}$, Kumiko Tahara ${ }^{1}$, Akihiko Takahashi ${ }^{1}$, Takeo Kudo ${ }^{2}$, Tadashi Motoori ${ }^{3}$, \\ Makoto Ohbu ${ }^{4}$, Hirobumi Kondo ${ }^{2}$ and Toshifumi Hibi ${ }^{5}$
}

\begin{abstract}
A 58-year-old Japanese woman presented with chronic fluctuating liver dysfunction with purpura. Raynaud's phenomenon had been diagnosed 4 years previously. At the initial examination, skin biopsy showed limited cutaneous systemic sclerosis (SSc). Laboratory investigations revealed liver dysfunction. Anti-nuclear antibodies, anti-mitochondria M2 antibody, anti-thyroglobulin antibody, and platelet-associated IgG were positive. Primary biliary cirrhosis (PBC) and autoimmune hepatitis (AIH) were diagnosed serologically, clinically and histologically. Immune thrombocytopenic purpura (ITP) was diagnosed by bone marrow puncture, clinical and laboratory findings, and Helicobacter pylori $\operatorname{IgG}$ was positive. She was treated with prednisolone $30 \mathrm{mg} /$ day, ursodeoxycholic acid $600 \mathrm{mg} / \mathrm{day}$, and a 7-day course of lansoprazole plus amoxicillin and clarithromycin. Thrombocytes increased rapidly and transaminase improved at day 7 . We report a rare case of PBC-AIH overlap syndrome with concurrent ITP and SSc which suggest the presence of shared genetic susceptibility factors in multiple autoimmune conditions including PBC, AIH, ITP and SSc.
\end{abstract}

Key words: primary biliary cirrhosis (PBC), autoimmune, hepatitis (AIH), systemic sclerosis (SSc), immune thrombocytopenic purpura (ITP)

(Inter Med 48: 2019-2023, 2009)

(DOI: 10.2169/internalmedicine.48.2687)

\section{Introduction}

Primary biliary cirrhosis (PBC) is a chronic cholestatic liver disease characterized by gradual destruction of the interlobular bile ducts leading to damage of the hepatocytes. The etiology remains unknown. The rate of disease progression is quite variable, but typically the disease is slowly progressive (1). Approximately half of PBC patients are affected by at least one additional autoimmune disease. A significant subgroup of patients (20\%) has multiple additional autoimmune conditions. These multiple autoimmune disease cases include combinations of Sjögren's syndrome, autoimmune thyroid disease, rheumatoid arthritis and scleroderma (2).

Autoimmune hepatitis (AIH) associated with connective tissue disorder is regarded to be rare. However, West et al (3) reviewed a case series of patients with AIH and reported that patients with AIH may be at increased risk for developing systemic CTD, while a review of literature reveals that systemic CTD may be at increased risk of developing AIH. The so-called "PBC-AIH overlap syndrome" has been defined as the concurrent manifestation of the main characteristics of the two conditions at the same time in the

\footnotetext{
${ }^{1}$ Division of Gastroenterology, Kitasato University, Kitasato Medical Center Hospital, Kitamoto, ${ }^{2}$ Division of Rheumatology, Department of Internal Medicine, Kitasato University, Kitasato Medical Center Hospital, Saitama, ${ }^{3}$ Division of Pathology, Kitasato University, Kitasato Medical Center Hospital, Saitama, ${ }^{4}$ Department of Pathology, School of Allied Health Sciences, Kitasato University, Sagamihara and ${ }^{5}$ Division of Gastroenterology, Department of Internal Medicine, School of Medicine, Keio University, Tokyo

Received for publication July 15, 2009; Accepted for publication August 26, 2009

Correspondence to Dr. Hiroaki Yokomori, yokomori@insti.kitasato-u.ac.jp
} 
same patient (4-6). In a comparative study, patients with PBC-AIH overlap syndrome presented with characteristic features of PBC (anti-mitochondrial M2 antibodies and bile duct damage compatible with $\mathrm{PBC}$ ), but a more hepatitic picture than a cohort of typical PBC patients (7). Here, we report a case of PBC-AIH overlap syndrome with cutaneous systemic sclerosis ( $\mathrm{SSc}$ ) and immune thrombocytopenic purpura (ITP).

\section{Case Report}

In 2009, a 58-year-old Japanese woman was admitted to a local hospital because of chronic fluctuating liver dysfunction. She had been diagnosed with Raynaud's phenomenon 4 years earlier. A skin biopsy from the left forearm showed irregularly thickened collagen bundles in the dermis and degenerated bundles of collagen fibers, with perivascular inflammatory infiltrate of mononuclear cells, and mildly reduced sweat and sebaceous glands (Fig. 1a). She fulfilled the criteria of systemic sclerosis (scleroderma) proposed by the American College of Rheumatology (8) and was classified as limited cutaneous SSc (lcSSc) according to the classification system proposed by LeRoy et al (9). She was transferred to our hospital for thorough investigation of liver dysfunction.

Her physical findings on admission to our hospital were as follows: body temperature, $36.8^{\circ} \mathrm{C}$; pulse, 69 beats/min and regular; blood pressure, $122 / 77 \mathrm{mmHg}$; respiratory rate $16 /$ min; no anemic sign at the conjunctiva palpebra; and icteric finding at conjunctiva bulbi. No flapping tremor or palmar erythema was observed. Superficial lymph nodes were not palpable. She had purpura on the left side of her leg. Physical examination of her hands revealed findings characteristic of scleroderma; namely, her skin was tight, smooth, glistening, and white (Fig. 1b). The lungs were normal on auscultation, chest radiograph, and electrocardiogram. The initial laboratory findings were as follows. Hematological data showed normal white blood cell count $(5.40 \times$ $10^{3} / \mu \mathrm{L}$; normal range, $3.3-9.0 \times 10^{3} / \mu \mathrm{L}$ ), slightly reduced red blood cell count $\left(3.68 \times 10^{4} / \mu \mathrm{L}\right.$; normal range, $4.30-5.70 \times 10^{4} /$ $\mu \mathrm{L})$, and reduced platelet count $\left(4.5 \times 10^{3} / \mu \mathrm{L}\right.$; normal range, $\left.140-340 \times 10^{3} / \mu \mathrm{L}\right)$. Erythrocyte sedimentation rate was 45 $\mathrm{mm} / \mathrm{h}$. Blood biochemical data were total bilirubin $1.3 \mathrm{mg} /$ dL (normal range: $0.2-1.2 \mathrm{mg} / \mathrm{dL}$ ), direct bilirubin $0.3 \mathrm{mg}$ / $\mathrm{dL}$ (normal range: $0.0-0.2 \mathrm{mg} / \mathrm{dL}$ ), aspartate aminotransferase (AST) 406 IU/L (normal range: 10-40 IU/L), alanine aminotransferase (ALT) $481 \mathrm{IU} / \mathrm{L}$ (normal range: 5-45 IU/ L), alkaline phosphatase 308 IU/L (normal range: 100-325 IU/L), $\gamma$-guanosine triphosphate $155 \mathrm{IU} / \mathrm{L}$ (normal range: < $30 \mathrm{IU} / \mathrm{L}$ ), blood urea nitrogen $10.6 \mathrm{mg} / \mathrm{dL}$ (normal range: 8.0-26 mg/dL), creatinine $1.2 \mathrm{mg} / \mathrm{dlL}$ (normal range: $0.47-$ $0.71 \mathrm{mg} / \mathrm{dL}$ ), $\operatorname{IgG} 2,020 \mathrm{mg} / \mathrm{dL}$ (normal range: $870-1,700$ $\mathrm{mg} / \mathrm{dL}$ ), and IgM $288 \mathrm{mg} / \mathrm{dL}$ (normal range: 46-260 mg/ dL). Hepatitis B surface antigen and anti-hepatitis C virus antibody were both negative. Anti-nuclear antibody (ANA titer 1 : 160, homogenous), anti-mitochondria M2 antibody

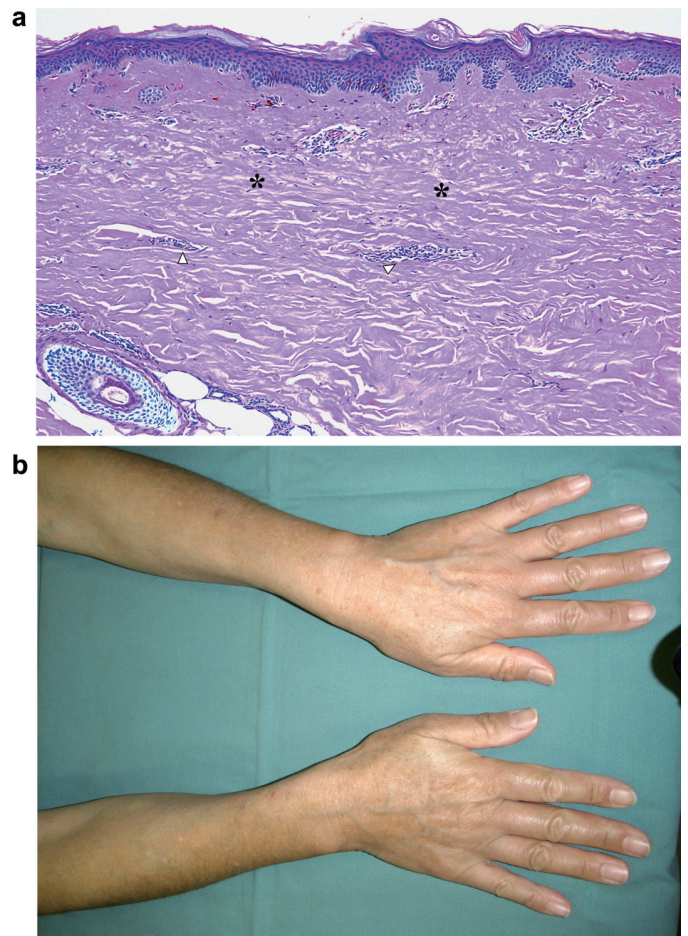

Figure 1. (a) Skin appearance and (b) dermal biopsy histological findings a: Skin appears tight, smooth, glistening, and white. b: Biopsy shows irregularly thickened collagen bundles (asterisks) in the dermis and degeneration of bundles of collagen fibers, with perivascular inflammatory infiltrate of mononuclear cells (arrowheads), and slightly decreased sweat or sebaceous gland.

(147; normal range $<7.0$ index), and anti-dsDNA antibody (26.5 IU/mL; normal range: $<20 \mathrm{IU} / \mathrm{mL}$ ) were positive. HLA-DR4 was positive. Rheumatoid factor (25 U; normal range: $<20 \mathrm{U}$ ) was positive. Rheumatoid arthritis hemagglutinin, anti-centromere antibody, anti-RNP antibody, anti-Scl70 antibody, and anti-Jo-1 antibody were all negative. With regard to thyroid function, thyroid stimulating hormone (1.254 $\mu \mathrm{IU} / \mathrm{mL}$; normal range: $0.436-3.8 \mu \mathrm{IU} / \mathrm{mL})$ and freethyroxin (1.14 ng/dL; normal range: 1.0-1.7 ng/dL) were within normal ranges. Anti-thyroglobulin antibody $(0.7 \mathrm{U} /$ $\mathrm{dL}$; normal range $<0.3 \mathrm{U} / \mathrm{mL}$ ) and platelet associated $\mathrm{IgG}$ (PAIgG: $50.5 \mathrm{ng} / 10^{7}$ cell; normal range: 5.0-25.0 ng/10 cell) were positive. Abdominal ultrasonography and computed tomography, conducted to examine the cause of hepatic dysfunction, detected no abdominal mass lesion. The patient also had no splenomegaly. Gastrointestinal fiberscopy revealed short segment Barrett's esophagus and gastric bleeding related toportal hypertensive gastropathy. Helicobacter pylori $\operatorname{IgG}(18 \mathrm{U} / \mathrm{mL}$; normal range: $<10 \mathrm{U} / \mathrm{mL})$ was positive. A liver biopsy showed epithelial damage in interlobular bile duct, intraepithelial lymphocytes and periductal lymphoplasmacyte infiltration, resembling chronic nonsuppurative destructive cholangitis (CNSDC) (Fig. 2a, b). PBC was diagnosed clinically and histologically according to the criteria proposed by the Japanese Joint Research Group for Autoimmune Hepatitis (10). Moreover, liver biopsy showed moderate to severe portal fibrosis with partial 
a
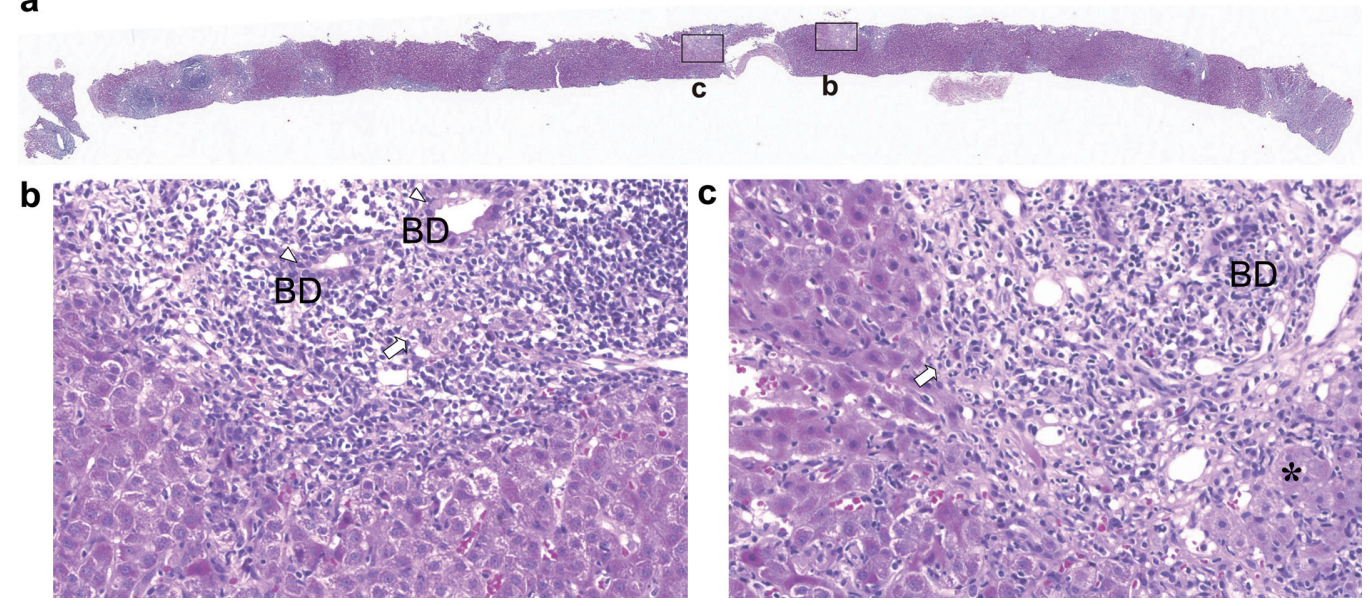

Figure 2. Histological findings of liver biopsy. a: Loupe photograph of the liver biopsy specimen. Low-magnification view $(\times 3.5)$ shows prominent portal inflammation distributed evenly throughout the portal tracts with mononuclear inflammation. b: Around an interlobular bile duct (BD), epithelial damage and intraepithelial lymphocyte infiltration are observed, resembling chronic nonsuppurative destructive cholangitis. A few epithelioid cells (arrow) are seen. Lymphocytes infiltrating the epithelium of an interlobular bile duct are observed (arrowheads). Magnification: $\times 200$, Hematoxylin and Eosin staining. c: Liver biopsy section shows a portal area heavily infiltrated with mononuclear inflammatory cells, interface hepatitis findings (arrow), rosette formation (asterisk), and hepatocyte necrosis surrounded by aggregates of lymphocytes. Magnification: $\times 200$, Hematoxylin and Eosin staining.

nodular formation in the parenchyma, interface hepatitis findings, and moderate to severe portal inflammation consisting of lymphocytes and plasma cells. Rosette arrangement of hepatocytes was commonly observed in the periportal zones (Fig. 2a, c). Inflammatory activity and the stage of fibrosis were classified as A2F2, according to the Inuyama classification (11). Cirrhosis was not evident. Autoimmune hepatitis was diagnosed provisionally. A final diagnosis of AIH was established on the basis of a positive antinuclear antibody test, high titers of serum globulin, no evidence of current hepatitis virus infection, histological findings, and a good response to corticosteroid therapy, all of which met the diagnostic criteria for type $1 \mathrm{AIH}$ (12). The patient had a post-treatment AIH score of 13 (probable AIH) according to the system proposed by the revised International Autoimmune Hepatitis Group (12). A bone marrow puncture showed normal cellular marrow with normal maturation of the erythrocytes, normal maturation of granulocytes and relatively increased megakaryocytes (Fig. 3). Immune thrombocytopenic purpura (ITP) was diagnosed from clinical and laboratory findings (13).

The patient received prednisolone $30 \mathrm{mg} /$ day; ursodeoxycholic acid $600 \mathrm{mg} /$ day; and a 7-day course consisting of lansoprazol $30 \mathrm{mg}$ twice a day (b.i.d.), amoxicillin $750 \mathrm{mg}$ and clarithromycin $200 \mathrm{mg}$ b.i.d. Platelet count increased slowly without further treatment from $4.5 \times 10^{3} / \mu \mathrm{L}$ at day 1 to $15.3 \times 10^{3} / \mu \mathrm{L}$ at day 7 . Transaminase levels began to decline after the administration of prednisolone for 3 days, and eventually were normalized in one month (Fig. 4).

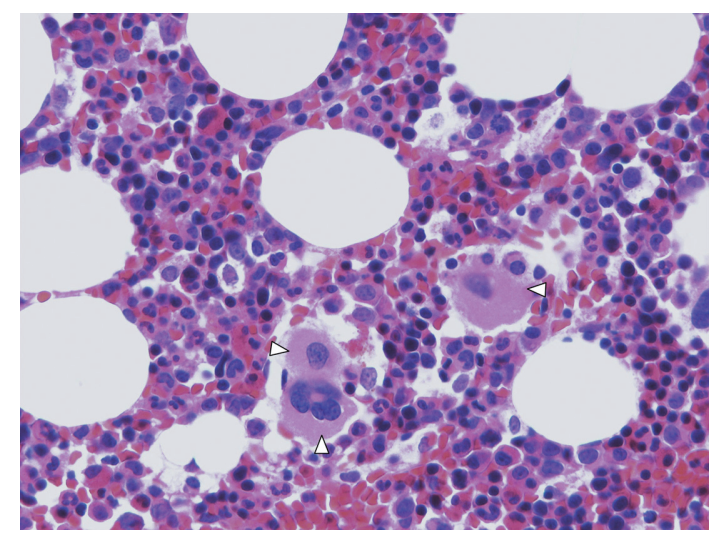

Figure 3. Bone marrow puncture findings. Hypercellular marrow with normal maturation of the erythrocytes, normal maturation of the granulocytes and relatively increased megakaryocytes are observed. Magnification: $\times 400$. Hematoxylin and Eosin staining. Arrowhead shows a megakaryocyte.

\section{Discussion}

The PBC-AIH overlap syndrome is a disease entity proposed by Popper and Schaffner (14) and has clinical features of both PBC and AIH. The histological characteristics of the disease are marked periportal necrosis, widespread bridging necrosis and CNSDC that are characteristic of PBC, together with a mixed finding of highly proliferated cholangioles and normal interlobular biliary tracts (15). The present patient, diagnosed with overlap syndrome, had high serum levels of ALT and IgM, showing mixed features of PBC and AIH.

Patients who have an overlap between $\mathrm{AIH}$ and PBC pose 


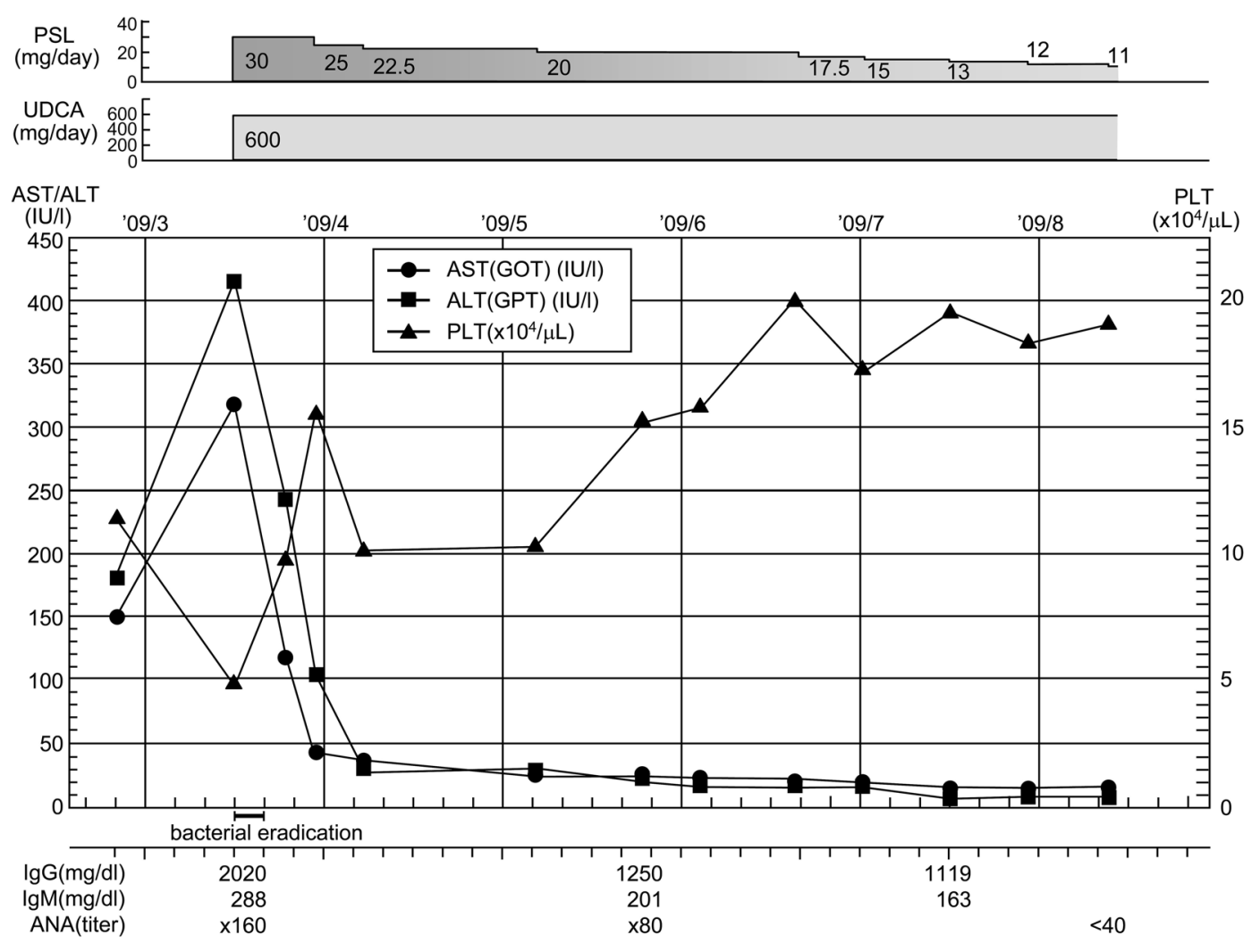

Figure 4. Clinical course

a major therapeutic dilemma. Ursodeoxycholic acid is a safe and life-extending therapy for most patients with PBC (16), whereas corticosteroids with or without azathioprine markedly improve survival in patients with AIH (17). Treatment with UDCA improves serum transaminase levels in patients with type $1 \mathrm{AIH}$, but change of histological activity after UDCA treatment has been controversial (18). Furthermore, the duration from treatment initiation to normalization of serum ALT levels is longer in UDCA treatment compared with corticosteroid treatment (19). Further studies are necessary to define the optimal therapeutic strategies in patients with overlapping $\mathrm{PBC}$ and $\mathrm{AIH}$.

The detection of AIH overlap syndromes appears to depend on the selection of specific diagnostic criteria. Uniform application of a systematic algorithm, therefore, is warranted to accurately determine the prevalence in different populations. The revised International Autoimmune Hepatitis Group (IAIHG) scoring system is a valuable tool for the diagnosis of classical AIH (12). However, the applicability of the IAIHG criteria for the diagnosis of variant forms of AIH is questionable $(20,21)$. The IAIHG score for the present case was 13, and is classified as probable AIH. In a study of PBC patients, probable AIH overlap was diagnosed in $19 \%$, with no case of definite AIH overlap identified using the revised IAHG scoring system (20).

The precise mechanism underlying ITP remains largely unknown. Immune dysregulation and the development of autoantibodies appear to play a major role (22). Autoantibodies are present in the plasma of patients with ITP. In approximately $50-70 \%$ of these patients, the antibodies recognize one or more platelet surface glycoproteins (GP) includ- ing GPIIb-IIIa, GPIb-IX and GPIa-II (22). In general, hypersplenism is suspected as a cause of thrombocytopenia in PBC. However, some patients with PBC exhibit autoantibodies; Dixon et al proposed the measurement of PAIgG in patients with $\mathrm{PBC}$ patient (23). As for the underlying mechanism of thrombocytopenia in PBC, a report has indicated that the glycoprotein complex of platelets and mitochondrial M2 antigen has similar amino acid sequences and they cross-react (24). ITP associated with PBC-AIH overlap syndrome is very rare, and our search of literature yielded only one case (25).

In Japan, the current standard first-line regimen for the eradication of $H$. pylori consists of a proton pump inhibitor, amoxicillin and clarithromycin for 1 week (26). The present patient received a 7-day course of triple therapy of lansoplazol, amoxicillin, and clarithromycin. There is growing evidence of an association between $H$. pylori eradication and platelet recovery in patients with ITP $(27,28)$. The evidence is strongest in Japan, where H. pylori eradication is now recommended as an initial treatment for $H$. pylori-positive ITP patients (29).

Although the prevalence of PBC concomitant with SSc is not clear, approximately $15 \%$ of patients with $\mathrm{PBC}$ have been reported to have SSc, mostly the variant of lcSSc (30). Concurrent autoimmune hepatitis and SSc is unusual. A Japanese patient with CREST syndrome and autoimmune hepatitis was reported (31). Marie et al (32) reported two patients with CREST variant of SSc, who developed autoimmune hepatitis. They proposed that since liver involvement may precede skin manifestations, the evaluation for SSc is appropriate when autoimmune hepatitis is noted. This evalu- 
ation should include clinical examination, antinuclear antibodies (especially for anticentromere antibodies) and nail fold capillaroscopy. The treatment of autoimmune hepatitis is difficult in patients with $1 \mathrm{cSSc}$ because patients receiving more than $15 \mathrm{mg}$ prednisone daily are at high risk of sclerodermal renal crisis (33). The present patient responded favorably to steroid therapy, both clinically and biochemically without untoward event.
We reported a rare case of PBC-AIH overlap syndrome with concurrent ITP and SSc which suggest the presence of shared genetic susceptibility factors in multiple autoimmune conditions including PBC, AIH, ITP and SSc.

\section{Acknowledgement}

We thank Dr. Hironobu Ura and Kazuto Hayashi for supplying the slide of skin biopsy in Fig. 1.

\section{References}

1. Kaplan MM, Gershwin ME. Primary biliary cirrhosis. N Engl J Med 353: 1261-1273, 2005.

2. Watt FE, James OF, Jones DE. Patterns of autoimmunity in primary biliary cirrhosis patients and their families: a populationbased cohort study. QJM 97: 397-406, 2004.

3. West M, Jasin HE, Medhekar S. The development of connective tissue diseases in patients with autoimmune hepatitis: a case series. Semin Arthritis Rheum 35: 344-348, 2006.

4. Czaja AJ. The variant forms of autoimmune hepatitis. Ann Intern Med 125: 588-598, 1996.

5. Poupon R. Autoimmune overlapping syndromes. Clin Liver Dis 7: 865-878, 2003.

6. Woodward J, Neuberger J. Autoimmune overlap syndromes. Hepatology 33: 994-1002, 2001.

7. Lohse AW, zum Buschenfelde KH, Franz B, Kanzler S, Gerken G, Dienes HP. Characterization of the overlap syndrome of primary biliary cirrhosis (PBC) and autoimmune hepatitis: evidence for it being a hepatitic form of PBC in genetically susceptible individuals. Hepatology 29: 1078-1084, 1999.

8. Preliminary criteria for the classification of systemic sclerosis (scleroderma). Subcommittee for scleroderma criteria of the American Rheumatism Association Diagnostic and Therapeutic Criteria Committee. Arthritis Rheum 23: 581-590, 1980.

9. LeRoy EC, Black C, Fleischmajer R, et al. Scleroderma (systemic sclerosis): classification, subsets and pathogenesis. J Rheumatol 15: 202-205, 1988.

10. Ohta Y. Diagnostic criteria for primary biliary cirrhosis. Acta Hepatol Jpn 33: 657, 1992 (in Japanese).

11. Ichida $F$, Tsuji $T$, Omata $M$, et al. New Inuyama classification; new criteria for histological assessment of chronic hepatitis. Int Hepatol Commun 6: 112-111, 1996.

12. Alvarez F, Berg PA, Bianchi FB, et al. International Autoimmune Hepatitis Group Report: review of criteria for diagnosis of autoimmune hepatitis. J Hepatol 31: 929-938, 1999.

13. Ikeda Y. The diagnosis and therapy of ITP. JJPA 19: 146-154, 2004 (in Japanese).

14. Popper H, Schaffner F. Nonsuppurative destructive chronic cholangitis and chronic hepatitis. Prog Liver Dis 13: 336-354, 1970.

15. Kloppel G, Seifert G, Lindner H, Dammermann R, Sack HJ, Berg PA. Histopathological features in mixed types of chronic aggressive hepatitis and primary biliary cirrhosis. Correlations of liver histology with mitochondrial antibodies of different specificity. Virchows Arch A Pathol Anat Histol 373: 143-160, 1977.

16. Poupon RE, Lindor KD, Cauch-Dudek K, Dickson ER, Poupon R, Heathcote EJ. Combined analysis of randomized controlled trials of ursodeoxycholic acid in primary biliary cirrhosis. Gastroenterology 113: 884-890, 1997.

17. Czaja AJ. Drug therapy in the management of type 1 autoimmune hepatitis. Drugs 57: 49-68, 1999.

18. Czaja AJ, Carpenter HA, Lindor KD. Ursodeoxycholic acid as adjunctive therapy for problematic type 1 autoimmune hepatitis: a randomized placebo-controlled treatment trial. Hepatology 30: 1381-1386, 1999.

19. Miyake Y, Iwasaki Y, Takaki A, Kobashi H, Sakaguchi K, Shira- tori Y. Clinical features of Japanese elderly patients with type 1 autoimmune hepatitis. Intern Med 46: 1945-1949, 2007.

20. Talwalkar JA, Keach JC, Angulo P, Lindor KD. Overlap of autoimmune hepatitis and primary biliary cirrhosis: an evaluation of a modified scoring system. Am J Gastroenterol 97: 1191-1207, 2002.

21. Farias AQ, Goncalves LL, Bittencourt PL, et al. Applicability of the IAIHG scoring system to the diagnosis of antimitochondrial/ anti-M2 seropositive variant form of autoimmune hepatitis. J Gastroenterol Hepatol 21: 887-893, 2006.

22. Chong BH, Ho SJ. Autoimmune thrombocytopenia: review. J Thromb Haemost 3: 1763-1772, 2005.

23. Dixon R, Rosse W, Ebbert L. Quantitative determination of antibody in idiopathic thrombocytopenic purpura. N Engl J Med 292: 230-236, 1975.

24. Panzer S, Penner E, Nelson PJ, Prochazka E, Benda H, Saurugger PN. Identification of the platelet glycoprotein IIb/IIIa complex as a target antigen in primary biliary cirrhosis-associated autoimmune thrombocytopenia. Evidence that platelet-reactive autoantibodies can also bind to the mitochondrial antigen M2. J Autoimmun 3: 473-483, 1990.

25. Arakawa Y, Amaki S, Miyakawa H, et al. PBC-AIH overlap syndrome with concomitant ITP and Hashimoto's disease with positivity for anti-centromere antibody. J Gastroenterol 39: 490-495, 2004.

26. Asaka M, Sugiyama T, Kato M, et al. A multicenter, double-blind study on triple therapy with lansoprazole, amoxicillin and clarithromycin for eradication of Helicobacter pylori in Japanese peptic ulcer patients. Helicobacter 6: 254-261, 2001.

27. Suzuki T, Matsushima M, Masui A, et al. Effect of Helicobacter pylori eradication in patients with chronic idiopathic thrombocytopenic purpura- a randomized controlled trial. Am J Gastroenterol 100: 1265-1270, 2005.

28. Suvajdzi N, Stankovi B, Artiko V, et al. Helicobacter pylori eradication can induce platelet recovery in chronic idiopathic thrombocytopenic purpura: prospective cohort study. Platelets 17: 227-230, 2006.

29. Fujimura K, Kuwana M, Kurata $\mathrm{Y}$, et al. Is eradication therapy useful as the first line of treatment in Helicobacter pylori-positive idiopathic thrombocytopenic purpura? Analysis of 207 eradicated chronic ITP cases in Japan. Int J Hematol 81: 162-168, 2005.

30. Abraham $S$, Begum $S$, Isenberg D. Hepatic manifestations of autoimmune rheumatic diseases. Ann Rheum Dis 63: 123-129, 2004.

31. Ishikawa M, Okada J, Shibuya A, Kondo H. CRST syndrome (calcinosis cutis, Raynaud's phenomenon, sclerodactyly, and telangiectasia) associated with autoimmune hepatitis. Intern Med 34: 6-9, 1995.

32. Marie I, Levesque H, Tranvouez JL, et al. Autoimmune hepatitis and systemic sclerosis: a new overlap syndrome? Rheumatology 40: 102-106, 2001.

33. Steen VD, Medsger TA Jr. Case-control study of cortico-steroids and other drugs that either precipitate or protect from the development of scleroderma renal crisis. Arthritis Rheum 41: 1613-1619, 1998.

(C) 2009 The Japanese Society of Internal Medicine

http://www.naika.or.jp/imindex.html 